\title{
Field Experiences in Breast Cancer Research among African American Women
}

\author{
Beverly Edwards, PhD \\ Fayetteville State University
}

\begin{abstract}
Despite many recent improvements in the health of Americans, substantial differences still exist among racial and ethnic groups. The frequently cited explanation for the disparity in health care for African Americans is lack of access to quality health care. Health care disparities are further complicated by increasing breast cancer rates of African American women without reduction of mortality rates. African American women have the highest breast cancer death rates. Despite all the gains that have been made in clinical, basic, and behavioral research, African American women continue to lag behind and remain disproportionately affected by this disease. In this qualitative pilot study, factors contributing to breast cancer risk in African American women were explored. Data was collected in four focus group sessions, where the study's participants were asked questions related to their perceptions of health status, lifestyles, and health care access. Cultural values had a major impact relating to food preferences, food preparation and seeking health care. These participant narratives provide insight into issues regarding their breast cancer risks and suggestions for future research.
\end{abstract}

Keywords: African American women, breast cancer, field experience, qualitative study

\section{INTRODUCTION}

African American women face an unequal breast cancer burden, due to known contributors, such as lack of access to health care, diet and sedentary lifestyle, as well as speculative causes as geographic location and hereditary (Duncan, Parrott, \& Silk, 2001). Researchers have also contended that African Americans suffer disproportionate effects or what some have termed a "cancer burden" in part due to African American women's (Greenberg \& Schneider, 1995; Sadler, Thomas, Dhanjal, Gebrekristos, 1998; Underwood, 1998.) higher mortality rate from breast cancer compared to European American women (Altpeter, Earp, \& Schopler, 1998; Breen \& Figueroa, 1996; Yood et al., 1999; Weiss et al., 1995). What are some of the factors that promote African American women's higher mortality rate from breast cancer when compared to women of other races or ethnicities? Are there specific factors that contribute to African American women being at higher risk for breast cancer? Limited research exists on African American women's perceptions and the impact of variables, such as lack of access to health care and low socioeconomic status on their breast cancer risk (Lipkus, Iden, Terrenoire, \& Feaganes, 1999). The researcher proposes to address this gap in understanding through an in-depth qualitative examination of African American women's perceptions of the impact of these contributors to their risks of breast cancer.

The researcher proposes to qualitatively investigate the environmental, social factors, personal behavioral correlates of health care access, and socioeconomic status for breast cancer risk in key informant focus groups of African American women in a state in the southern region of the United States (U.S.). This method of data collection provides African American women the opportunity to voice their concerns and perspectives about lifestyles, socioeconomic status and 
health. It is expected that these narratives will provide valuable information for a better understanding of this issue, but possible insights to aid in the development of effective interventions to address this major public health issue. These narratives reveal lack of access to health care and low socioeconomic status as contributors to access and the use of mammography, which in turn leads to delays in the diagnosis of breast cancer. This paper presents the findings from four of these key informant focus groups.

\section{LITERATURE REVIEW}

Differences in mortality and five-year survival rates for European American and African American women due to breast cancer have been identified in previous research (Altpeter et al., 1998; Breen \& Figueroa, 1996; Underwood, 1998; Weiss et al., 1995; Yood et al., 1999). According to Surveillance, Epidemiology, and End Results (SEER) (Ries, Kosary, Hankery, Miller, Harras, \& Edwards, 1997), there are significant differences in the five-year survival rates for African American women (70.0 per 100,000) and all other races of women (84.2 per 100,000). Although a number of reasons for the differences have been identified, some variables have been identified consistently across studies. These include low socioeconomic status (SES), lack of access to health care, low screening rates, and later stage of disease at initial detection. Much of the research published has focused on the later stage of the disease at initial detection, attributing this to a lack of access to health care (Altpeter et al., 1998; Breen \& Figueroa, 1996; Forte, 1995; Mandelbaltt, Yabroff, \& Kerner, 1999; Shaw, 1996: Underwood, 1998; Weiss et al., 1995.). Lack of access to health care contributes to a decreased availability and use of mammography, as well as diagnosis at later stages of breast cancer. Both of these variables are associated with increased breast cancer mortality rates. Although national statistics reveal breast cancer mortality declined 3.4\% between 1995 and 1998, the decline was much greater among white women than among black women. It has been found that African American women with breast cancer are less likely than Caucasian women to survive 5 years at a rate of $72 \%$ vs. 87\%. (Agency for Health Care Research and Quality (AHRQ), 2002).

The cancer death rate among African Americans (both men and women) is about $35 \%$ higher than it is for Caucasians. Although substantial progress has been made in diagnosing and treating breast cancer, it continues to take a heavy toll, particularly among black women. The numbers are startling, Caucasian women are 12-29\% more likely to be diagnosed with breast cancer yet African American women are 28\% more likely than Caucasian women to die from the disease. Breast cancer survival rates rose slightly among Caucasian women over the past two decades, but during this time survival rates declined for African American women. This disparity is even more pronounced when we consider that the 5-year breast cancer survival rate is only 69\% for African American women, as compared with $85 \%$ for Caucasian women (AHRQ, 2002).

To capture a current picture of African American health care experiences, out of a sample size of 6,722 by the Commonwealth Fund 2001 Health Care Quality Survey 1,037 Americans identified themselves as African American or black. Across all ages and even more notably by age 50, African Americans are living with higher rates of chronic diseases. Nearly one-third of African Americans ages 18 to 64 were uninsured during the year (30\%), compared with one of five $(20 \%)$ nonelderly white adults. Although rates of employment varied little, African American nonelderly adults are also notably less likely to have job-based insurance (59\% vs. $70 \%$ Caucasian) and more likely to rely on public programs (11\% African American vs. 4\% Caucasian). Lack of medical insurance reflects racial income disparities: 50 percent of African Americans compared with 30 percent of Caucasians report annual income at poverty or near poverty levels (below 200\% of poverty). While having a doctor familiar with your health 
concerns has been identified as a key factor to obtaining quality care, 28 percent of African American adults say they have no regular doctor, compared with 19 percent of Caucasians, African Americans were more likely to self-report that hospital emergency rooms, clinics, or nowhere as their usual source of care (22\% of African American adults compared with $5 \%$ of Caucasians). Twenty two percent of African Americans compared with 15 percent of Caucasians report very little or no choice in where they go for care. Lack of a regular doctor greatly undermines quality of care. African Americans who do not have a regular doctor are less likely to receive preventive services such as physical exams, and report they are less confident in and satisfied with their care, and are more likely to report communication problems (Collins, Tenney, \& Hughes, 2002, pp. 1-2).

Several studies have shown that access to health care is associated with improved health outcomes. However, experiences with health care services delivery appear to differ significantly and are impacted greatly by intersecting effects of race, ethnicity, and gender. Moreover, overall health status among racial and ethnic minority groups and women underscores significant problems in access to health care. (Franks, Gold, \& Clancy, 1996). While health care access and other societal contributors are important to address in health policy efforts to reduce the incidence of breast cancer among African American women, the picture appears to be far more complex. Wojcik, Spinks, and Optenberg (1997) examined European American women and African American women treated in U.S. military health care facilities with equal access to health care and treatment options. They found that access to health care and treatment options decreased the mortality rate for African American women military beneficiaries (24.77\%) when compared to other African American women (34.2\%).

This study is very timely in that it attempts to address this public health concern in a population disproportionately affected by the consequences of lack of health care access and socioeconomic status for breast cancer risk among African American women.

\section{STUDY DESIGN AND METHODS}

A qualitative research design was used for this particular study. Qualitative methods are particularly useful tools when the research task involves generating meaning for the lived experiences of underrepresented groups (Creswell, 1998; Saleeby, 1994). Accordingly, in order to better understand the experiences of the African American women who participated in this study it required the use of ethnographic methods that allowed these women to tell their own stories in their own words. To better understand the complexities of health care access and socioeconomic status in African American women in this state in the southern region of the United States ethnographic exploration allowed for African American women to report concerns, attitudes, and everyday experiences with social factors, environmental, and personal behavioral issues related to health care access and socioeconomic status for breast cancer risk in small focus group discussion sessions.

\section{SAMPLE}

The sample was composed of 40 African American women 18 years of age or older who were residents in this southern U.S. state. Participants were in a snowball sampling method from a number of locations, including a university, a college, local churches, and other locations in the community to obtain representation of African American women from various backgrounds and socioeconomic status. Flyers were posted in a number of locations on campus, on church bulletin boards, and in the community inviting African American women to participate by 
contacting the researcher by phone. Participants were offered a $\$ 20.00$ stipend as an incentive for participation in the focus groups. The largest respondent age group was 26-35 (35\%) with representation among other groups listed as follows: 18-25 years (10\%), 36-45 years (25\%), 46-55 years (20\%), and 56-65 years $(10 \%)$. The majority of respondents were single $(43 \%)$ and reported an average income of $\$ 15,000$ - \$30,000 per year. Seventeen women (43\%) reported they were single and living alone. Twenty women (50\%) reported their health was very good. Thirty four women (85\%) reported they had some kind of health care coverage. Thirty three women (83\%) reported they had access to health care insurance. Table 1 provides more detailed demographic information of the respondents.

Table 1

Demographic Characteristics of the Respondents

\begin{tabular}{|lcc|}
\hline \multicolumn{1}{|c|}{ Racial Identity/Ethnicity } & Frequency & Percentage of Sample $(\mathrm{N}=40)$ \\
\hline African American & & \\
\hline Age & 4 & 10 \\
\hline $18-25$ & 14 & 35 \\
\hline $26-35$ & 10 & 25 \\
\hline $36-45$ & 8 & 20 \\
\hline $46-55$ & 4 & 10 \\
\hline $56-65$ & & \\
\hline Relationship Status & 17 & 43 \\
\hline Single & 10 & 25 \\
\hline Married & 7 & 17 \\
\hline Single/living with significant & & \\
\hline Other & 6 & 15 \\
\hline Divorced/Separated & & \\
\hline Family Income & 7 & 17 \\
\hline Below $\$ 15,000$ & 16 & 40 \\
\hline$\$ 15,000-\$ 30,000$ & 13 & 33 \\
\hline$\$ 31,000-\$ 45,000$ & 3 & 7 \\
\hline$\$ 46,000-\$ 60,000$ & 1 & 3 \\
\hline Above $\$ 60,000$ & & \\
\hline
\end{tabular}

\section{PROCEDURES}

Respondents who agreed to participate in the study were randomly assigned a date, time and location to participate in one of the four focus groups. The focus group facilitator began the sessions by requesting all participants read and sign an informed consent. Participants were then asked to complete a demographic survey that contained questions about their individual health care concerns, lifestyle and culture. This information was analyzed using descriptive statistics to provide a description of sample characteristics. Qualitative data was collected from 4 focus groups comprising 10 participants each in a structured interview process where participants were asked questions regarding their perceptions of personal behavior, social factors, the environment, health care access, and socioeconomic status. Sample interview questions include: What type of lifestyle do you have including diet and exercise? What are some of the health concerns that you have for yourself as an African American woman? Are there things about African American culture and communities that you feel promote higher risks of breast cancer in African American women? and Do you feel that socioeconomic status and health care access are major contributors to African American being at higher risk for breast cancer when compared to women of other races/ethnicities? Each focus group session 
lasted approximately 2 hours. The focus group sessions were audio and video taped for transcription purposes to insure the accuracy and each transcription was checked against the original and audio tape. In this method, if any inaccuracies were detected, corrections were made in the transcription and the interview was entered into a qualitative data analysis program to detect themes related to environmental, social factors, and personal behavioral issues related to health care access and socioeconomic status for breast cancer risk within the focus group discussions. Data analysis was facilitated through the use of NVIVO (QSR, 1999) a software package for managing and analyzing qualitative data.

\section{RESULTS AND DISCUSSION}

When the transcripts were reviewed, there were major themes within the participant's comments on lifestyle and health care access. Participants perceived themselves in need of a healthier lifestyle and to visit their physician on a regular basis. Another emerging theme that came out of the focus group discussions was the African American women's lack of a regular doctor which can greatly undermine the quality of care.

\section{Health Status}

Table 2 illustrates the findings regarding the questions related to health care and access in this study. The majority of respondents (50\%) reported that they perceived their health as very good. Sixty five percent (65\%) of the respondents did not consider themselves at risk for breast cancer and eighty two percent (82\%) of respondents reported that they had access to health care insurance with twenty three percent (23\%) of respondents reporting that they have more than one personal doctor or health care provider. Thirty percent $(30 \%)$ of the respondents reported that there was a time in the past twelve months when they needed to see a doctor but could not because of cost while forty three percent (43\%) of the respondents reported they had never had a mammogram. 
Table 2

Self Perception and Health Status among Respondents in Sample

\begin{tabular}{|lc|}
\hline \multicolumn{2}{|c|}{ Percentage of Sample (N=40) } \\
\hline Health status-self perception & 7 \\
\hline Excellent & 50 \\
\hline Very good & 35 \\
\hline Good & 8 \\
\hline Fair & 85 \\
\hline Health care coverage & 15 \\
\hline (health insurance, HMO, Medicare) \\
\hline Yes \\
\hline No & 82 \\
\hline Access to health care insurance \\
\hline Yes & 18 \\
\hline No & 68 \\
\hline Participation in any physical activities or \\
\hline exercises (jogging, walking, aerobics, etc.) \\
\hline Yes & 32 \\
\hline No & 35 \\
\hline Feel they are at risk for breast cancer \\
\hline Yes \\
\hline No \\
\hline Had a mammogram \\
\hline Yes \\
\hline No \\
\hline \multicolumn{2}{|l|}{} \\
\hline
\end{tabular}

Concern in finding a personal care physician or health care provider who was sincere was the most frequently given reason for one of the health concerns that the participants had for themselves as African American women. Another concern reported by the participants was not being able to afford health care if they were really sick. One participant stated "I worry as I age that I'm not going to be able to afford it. The cost of insurance is extremely high. My fear is the cost of medical treatment."

\section{Social Factors to Breast Cancer Risks among African American Women}

When asked questions about African American culture and communities that promoted higher risks of breast cancer in African American women, issues relating to unhealthy eating, food preparation and food preferences emerged as themes. Statements such as "I learned how to cook fried foods and fat back from my mom." "There's a lot of fatty meats that you can get for a cheaper price." "We cook with a lot of salt and things saturated with grease." "We eat out more because of convenience." are examples of this content. Participants indicated that culturally, many African American women do not see a reason or a need to go to the doctor if they're really not hurting. This viewpoint was supported by statements such as, "Part of our belief is that God will heal us." "Our religious beliefs and how we practice sometimes plays a real big part in shaping how we deal with our health." Participants felt that African American women are so busy providing for everybody else in their house hold that they neglect themselves. Others reported that African American women are not being educated in their communities about specific cancer risks. 
When asked to describe what it means to be an African American woman in this southern U.S. state, the participants indicated that they were taught to be caretakers, independent, and strong. One participant stated "I came to find that we're very resilient because no matter what the circumstances are we're always able to pull something out of the bag and make it work no matter what." Participants stated that they were taught spiritual and moral values and to be proud black women and education is important. When asked what are some of the factors that they believed contribute to AA women being disproportionately at risk for cancer when compared to women of other races/ethnicities, the participants responded by stating "We don't have accessible health care in our community and if we get sick we have to travel outside of our community therefore we have to have transportation to get there." Participants also stated that their families don't talk about breast cancer risks openly and reported that they accessed emergency care because they really did not have their own private doctors.

\section{Personal Behavioral Factors to Breast Cancer Risks among African American Women}

When asked to discuss their lifestyles, participants indicated that they have a sedentary lifestyle and are not consistent with exercise. One participant stated, “I don't eat right because I'm on the go all the time." Another participant indicated that she was not consistent with exercise. She stated, "And then I exercise now but I may go two months without exercising." Additionally, participants indicated that they were emotional eaters, had poor eating habits and eat when depressed. One participant explained "I figured out that it has a lot to do with worrying which turns into what I found out was depression that we don't like to admit as black women." This statement about the importance of food and the many roles it plays in the lives of this sample of African American women is powerful and indicative of how food is involved across the emotional spectrum from depression, to happiness, to stress.

\section{CONCLUSION}

The findings in this study indicate that African American women have culturally defined views about breast cancer risks that significantly influence their attitudes about breast cancer. A key finding of interest is that these women report that women are more afraid of what they're going to be told when they go to the doctor. This indicates that fear is the primary motivator for women not going to the doctor for regular checkups. The majority of respondents in this sample reported they are also fearful of not being able to afford the medical care. Some of the participants reported that they had access to health care but they felt discouraged to go when access meant sitting in public health departments' waiting rooms for four or five hours before they are able to see a doctor. Other respondents in the study discussed not being educated about breast cancer risk and not communicating with each other about the issue.

Although this is a very limited sample, the implications of these findings underscore the necessity of conducting additional research on this topic to better address population specific health care disparities for African American women and breast cancer risks. Moreover, few studies have considered race as a central factor in comprehensive breast cancer risk assessment. Further, research provides evidence of the need for the development of culturally specific interventions in order to assist African American women who are at risk for breast cancer. In order to reduce risk and rates of breast cancer, additional research is needed to ascertain the relative importance of cultural and psychosocial contributors to the development of negative or complicating attitudes towards breast cancer. Specifically, Sussner, Edwards, Thompson, Jandorf, Kwate, Forman, Brown, Kapil-Pair, Bovbjerg, Schwartz, and Valdimarsdottir (2011) suggest that such culturally-based interventions which are grounded 
in a greater understanding of how cultural background may influence beliefs, attitudes and reactions to hereditary will influence the design of more culturally sensitive practices. Finally, professional practitioners and counselors may be able to use this information to ensure that decisions related to hereditary testing for cancer risks are fully informed and culturally sensitive and appropriate within African American women.

\section{References}

Altpeter, M., Earp, J. A. L., \& Schopler, J. H. (1998). Promoting breast cancer screening in rural, African American communities: The science and art of community health promotion. Health \& Social Work, 23, 104-115.

Breen, N., \& Figeroa, J. B. (1996). Stage of breast and cervical cancer. American Journal of Preventive Medicine, 12(5), 319-326.

Collins, K. S., Tenney, K., \& Hughes, D. L. (2002, March). Quality of health care for African Americans. Retrieved from http://www.commonwealthfund.org/.

Creswell, J. W. (1998). Qualitative inquiry and research design: Choosing among five traditions. Thousand Oaks, CA: Sage.

Duncan, V. J., Parrott, R. L., \& Silk, Kami J. (2001). African American women's perceptions of the role of genetics in breast cancer risk. American Journal of Health Studies, 17(2), 50-59.

Forte, D. A. (1995). Community-based breast cancer intervention program for older African American women in beauty salons. Public Health Reports, 110, 179-183.

Greenberg, M., \& Schneider, D. (1995). The cancer burden of southern-born African Americans: Analysis of a social-geographic legacy. Milbank Quarterly, 73(4), 599-620.

Lipkus, I. M., Iden, D., Terrenoire, J., \& Feaganes, J. R. (1999). Relationships among breast cancer concern, risk perceptions, and interest in genetic testing for breast cancer susceptibility among African American women with and without a family history of breast cancer. Cancer Epidemiology, Biomarkers, \& Prevention, 8, 533-539.

Mandeblatt, J. S., Yabroff, K. R., \& Kerner, J. F. (1999). Equitable access to cancer services: A review of barriers to quality care. Cancer, 86, 2379-2390.

Ries, L. G., Kosary, C. L., Hankey, B. F., Miller, B. A., Harras, A., Edwards, B. K. (1997).

SEER Cancer Statistics Review, 1973-1994. NIH pub. no. 97-2789. Bethesda, MD: National Cancer Institute.

Sadler, G. R., Thomas, A. G., Dhanjal, S. K., Gebrekristos, B., \& Wright, F. A. (1998). Breast cancer screening adherence in African American women: Black cosmetologists promoting health. Cancer, 83, 1836-1839.

Saleeby, D. (1994). Culture, theory, and narrative: Intersection of meanings in practice. Social Work, 39(4), 351359.

Shaw, M. W. (1996). Influencing breast cancer knowledge, beliefs, and screening practices among Black women. Journal of Wellness Perspectives, 12(4), 180-187.

Sussner, K. M., Edwards, T. A., Thompson, H. S., Jandorf, L., Kwate, N. O., Forman, A., Brown, K., Kapil-Pair, N., Bovbjerg, D. H., Schwartz, M. D., \& Valdimarsdottir, H. B. (2011). Ethnic, racial and cultural identity and perceived benefits and barriers related to genetic testing for breast cancer among at-risk women of African descent in New York City. Public Health Genomics, 14, 356-370.

Underwood, S. M. (1998). Reducing the cancer burden among African Americans: A call to arms. Cancer, 83, 1877-1884.

Weiss, S. E., Tamer, P. L. Ahmed, S., Brower, S. T., Brusco, C., Bossolt, K., Amberson, J. B., \& Bratton, J. (1995). Ethnic differences in risk and prognostic factors for breast cancer. Cancer, 76, 268-274.

Wojcik, B. E., Spinks, M. K., \& Optenberg, S. A. (1998). Breast carcinoma survival analysis for African American and White women in an equal-access health care system. Cancer, 82, 1310-1318.

Yood, M. U., Johnson, C. C., Blount, A., Abrams, J., Wolman, E., McCarthy, B. D., Raju, U., Nathanson, D. S., Worsham, M., \& Wolman, S. R. (1999). Race and differences in breast cancer survival in a managed care population. Journal of the National Cancer Institute, 91, 1487-1491. 